\title{
Costs of a contact screening activity in a neonatal intensive care unit
}

\author{
Kate E. Fisher ${ }^{\mathrm{A}}$, Nicole F. Cook ${ }^{\mathrm{B}}$ \\ and Guy B. Marks \\ ${ }^{\mathrm{A}}$ Department of Community Paediatrics, South Western Sydney \\ Local Health District \\ ${ }^{\mathrm{B}}$ Community Health/Paediatric Ambulatory Care Services, South \\ Western Sydney Local Health District \\ ${ }^{\mathrm{C}}$ Department of Respiratory Medicine, Liverpool Hospital
}

In March 2011, a medical officer working in a metropolitan New South Wales (NSW) neonatal intensive care unit (NICU) was diagnosed with active pulmonary tuberculosis (TB). He had immigrated to Australia from a high-incidence country 2 years earlier with negative pre-immigration screening. Occupational TB screening prior to commencing employment at the hospital, conducted in line with NSW Health policy, ${ }^{1}$ was also negative. The man was screened again, also in line with the NSW Health policy, after he displayed TB symptoms (i.e. a 6-month history of nonproductive cough and weight loss), and was smear-positive on induced sputum. Mycobacterium tuberculosis was confirmed on polymerase chain reaction, fully sensitive to standard anti-TB therapy.

The index case worked full-time in the NICU during his infectious period from December 2010 to March 2011. As neonates are at higher risk than older children or adults of developing severe and potentially fatal disease soon after infection with $\mathrm{TB}$, it was decided to immediately offer screening to all exposed neonates and others who were potentially exposed rather than implementing a staged response. 2,3 The outcome of this investigation is being reported elsewhere. ${ }^{4}$ Here we describe the costs of the investigation.

\section{Contact investigation}

All 125 neonates, 165 of their relatives and 122 health care workers identified from medical records and departmental rosters as having had contact with the index case during his infectious period were offered screening (Table 1).

Ninety-six neonates were followed up at the hospital, 15 were followed up in other Local Health Districts, nine had died of unrelated causes and five failed to attend. Exposed neonates received a tuberculin skin test (TST) at 3 months corrected age; TSTs have been shown to be unreliable prior to this age. ${ }^{5-7}$ Neonates who were too young to be screened were commenced on isoniazid prophylaxis until they reached 3 months corrected age.
Relatives and health care workers were offered TST screening at the Chest Clinic. A reaction of $\geq 10 \mathrm{~mm}$ (or $\geq 15 \mathrm{~mm}$ with a Bacille Calmette-Guérin (BCG) scar) was defined as a positive TST.

Data on exposure and outcomes were collected from clinical records and departmental rosters. None of the 100 neonates screened (89 in the hospital and 11 in other Local Health Districts) had positive TSTs. ${ }^{4}$ Fifty-one of $152(34 \%)$ relatives and nine of $120(8 \%)$ health care workers screened had positive TSTs. ${ }^{4}$ All subsequently had negative chest X-rays, sputum cultures and/or interferongamma release assays (IGRAs). All positive TSTs were associated with origin from a high-incidence country and/ or previous $\mathrm{BCG}$ vaccination. No adult contacts were treated for TB infection or disease.

\section{Resources used}

The time spent on this investigation by nurses, doctors and pharmacists was obtained from rosters and interviews with staff. Salaries were calculated using 2011 NSW Health awards. ${ }^{8-10}$ Travel costs were estimated using National Roads and Motorists' Association costs for a mediumsized vehicle. ${ }^{11}$ The costs of laboratory tests and radiology were obtained from the Medicare Benefits Schedule (items 58503 and 69327), and hospital radiology and pathology departments. $^{12}$ The hospital pharmacy provided information on the cost of isoniazid. The cost of consumables was calculated from clinic invoices. Car parking and postage costs were obtained from the investigation cost centre. All costs presented are therefore estimates (Table 2).

Neonates were screened by Chest Clinic registered nurses. Neonates on isoniazid were also monitored in medical review clinics. Chest Clinic and infection control clinical nurse consultants also attended the neonatal clinics. The time nurses spent on neonatal screening cost $\$ 24489 .^{8}$ Chest Clinic registered nurses also conducted the relative and health care worker screening at a cost of $\$ 14913 .^{8}$

Neonatologists and paediatricians reviewed the neonates at the screening and medical review clinics at a cost of $\$ 19059 .^{9}$ Respiratory physicians interviewed and counselled the families of exposed neonates at the initial screening clinics at a cost of $\$ 9148 .{ }^{9}$

The estimated $910 \mathrm{~km}$ travelled for screening home visits and medication delivery cost approximately $\$ 682 .^{11}$ 
Table 1. South Western Sydney Local Health District neonatal intensive care unit tuberculosis contact investigation: exposed contact demographics and screening outcomes, 2011

\begin{tabular}{lllr}
\hline & Neonates & Relatives & Health care workers \\
\hline Number exposed & 125 & 165 & 122 \\
Mean age at start of investigation & 2.1 weeks corrected & 32.5 years & 40.1 years \\
& $(95 \%$ Cl: $1.0-3.1)$ & $(95 \%$ Cl: $31.0-33.4)$ & $(95 \%$ Cl: $38.0-42.1)$ \\
Gender & & & $12(10 \%)$ \\
$\quad$ Male & $70(56 \%)$ & $72(44 \%)$ & $110(90 \%)$ \\
Female & $55(44 \%)$ & $93(56 \%)$ & $54(44 \%)$ \\
Born overseas & $0(0 \%)$ & $72(44 \%)$ & $65(53 \%)$ \\
Previous BCG & $0(0 \%)$ & $23(14 \%)$ & $0(0 \%)$ \\
Previous known TB & $0(0 \%)$ & $2(1 \%)$ & $0(0 \%)$ \\
Received isoniazid prophylaxis & Hospital clinic: $66 / 89(74 \%)$ & $0(0 \%)$ & $9 / 120(8 \%)$ \\
Positive TST during investigation & Hospital clinic: $0 / 89(0 \%)$ & $51 / 152(34 \%)$ & \\
\end{tabular}

BCG: Bacille Calmette-Guérin; Cl: confidence interval; TST: tuberculin skin test.

Table 2. Estimated costs of the neonatal intensive care unit tuberculosis contact investigation for the contacts screened in the South Western Sydney Local Health District, 2011

\section{Category}

Nurses' time (99 days of chest clinic RN time, 11 days of chest clinic CNC time, 10 days of infection control

CNC time, 2 weeks of neonatology RN time)

Doctors' time (25 days of neonatologist and paediatrician time, 12 days of respiratory physician time)

Investigation coordination (23 weeks of CNC time)

Home visits (Travel costs over $910 \mathrm{~km}$ and nursing time of $10 \mathrm{RN}$ hours and 12 CNC hours)

Screening tests (220 CXRs, 487 TSTs, 10 IGRAs, $2 \times 3$ sputum smear/cultures)

Isoniazid prophylaxis (4425 days of isoniazid solution and 69 hours of pharmacist time)

Other (consumables, car parking, postage)

Total estimated cost

NB: Costs associated with screening neonates living in other Local Health Districts are not included in this estimate.

CNC: clinical nurse consultant; CXR: chest X-ray; IGRA: interferon-gamma release assay; RN: registered nurse; TST: tuberculin skin test.

\section{Estimated cost}

$\$ 39402$

$\$ 28207$

$\$ 40970$

$\$ 1653$

$\$ 12450$

$\$ 2658$

$\$ 3090$

$\$ 128430$
Nurses' time spent conducting home visits cost an additional $\$ 971 .{ }^{8}$ A clinical nurse consultant was appointed to coordinate the investigation over 23 weeks at a cost of $\$ 40970 .{ }^{8}$ Screening tests conducted included chest X-rays (220 performed for a total of \$10373), TSTs (487 performed for \$1406), IGRAs (10 performed for \$500) and sputum smears/cultures (two contacts with three specimens each for $\$ 171){ }^{12}$

The 66 neonates commenced on prophylaxis received a total of 4425 isoniazid treatment days. At an average dose of $50 \mathrm{mg} /$ day the isoniazid solution cost $\$ 167$, with no additional cost passed onto the families. Manufacturing and dispensing the isoniazid solution took the hospital pharmacists approximately 69 hours, at a cost of $\$ 2491 .^{10}$

Additional costs included consumables (\$1034), parking for families (\$1826) and postage for letters sent to families and health care providers (approximately \$230).
There are few neonatal nosocomial TB exposure investigations in the published literature, and little evidence on their cost. ${ }^{2,5,13-16}$ We estimate the total cost to the hospital of this screening investigation was $\$ 128430$. This may underestimate the true cost as some items could not be quantified, including the cost of screening the 15 neonates and their relatives who attended other facilities for screening, the cost to families of travel to the clinics and pharmacy, and the investigation hotline. The time spent by hospital executives, the media unit, the NSW Ministry of Health and Public Health Unit staff is also not included in this estimate.

Indirect costs that are difficult to quantify include the cost of a parent's time away from work and usual duties. The opportunity cost to other departments of scarce health care resources including health care worker time, clinic space and tests used for the investigation is significant but difficult to quantify. 
The potentially substantial costs of screening investigations are not always included within health service budgets. This episode highlights the importance of including funds for screening within the public health or prevention components of budgets to accommodate such events.

Intangible costs including the anxiety and stress caused to families from the potential infection of their neonate, as well as to exposed health care workers and relatives, are considerable but not readily expressed in dollar terms.

\section{Conclusion}

There is a paucity of evidence around neonatal nosocomial TB exposure events, making it difficult to plan an appropriate response. In the absence of information it is difficult to justify not taking a precautionary approach when neonates are involved, but alternative approaches could be considered. Rather than screening all contacts as in this investigation, after careful risk assessment screening could initially be restricted to those with the highest exposure and extended to those at lower risk only if cases are detected. If no evidence of transmission was detected in the most highly-exposed contacts, further unnecessary screening of contacts at lower risk would be prevented, resulting in potential cost savings.

No evidence of transmission was detected in this and previous similar investigations; the risk of nosocomial transmission in the NICU setting appears to be low. ${ }^{2-7,13-17}$ This may add confidence that a staged approach for future TB screening activities in neonatal settings can be a safe and cost-effective alternative to initially screening all potentially exposed neonates.

\section{Acknowledgments}

The authors wish to acknowledge the hard work of staff from the South Western Sydney Local Health District Chest Clinic, NICU, Departments of Paediatrics and Community Paediatrics, and the Sydney and South Western Sydney Public Health Unit during the investigation.

\section{References}

1. NSW Health. Occupational Assessment, Screening and Vaccination Against Specified Infectious Diseases. Policy Directive PD2011_005. Available at: http://www.health.nsw.gov.au/ policies/pd/2011/pdf/PD2011_005.pdf (Cited 25 June 2013).

2. Ohno H, Ikegami Y, Kishida K, Yamamoto Y, Ikeda N, Taniguchi $\mathrm{T}$ et al. A contact investigation of the transmission of Mycobacterium tuberculosis from a nurse working in a newborn nursery and maternity ward. $J$ Infect Chemother 2008; 14(1): 66-71. doi:10.1007/s10156-007-0565-0
3. Nivin B, Nicholas P, Gayer M, Frieden TR, Fujiwara PI. A continuing outbreak of multidrug-resistant tuberculosis, with transmission in a hospital nursery. Clin Infect Dis 1998; 26(2): 303-7. doi:10.1086/516296

4. Fisher KE, Guaran R, Stack J, Simpson S, Krause W, For KD et al. Contact investigation of neonates, relatives and healthcare workers following nosocomial tuberculosis exposure. Infect Control Hosp Epidemiol (in press)

5. Light IJ, Saidleman M, Sutherland JM. Management of newborns after nursery exposure to tuberculosis. Am Rev Respir Dis 1974; 109(4): 415-9.

6. Lee LH, LeVea CM, Graman PS. Congenital tuberculosis in a neonatal intensive care unit: case report, epidemiological investigation, and management of exposures. Clin Infect Dis 1998; 27(3): 474-7. doi:10.1086/514690

7. Kim KI, Lee JW, Park JH, Kim SY, Park HJ, Choi PJ et al. Pulmonary tuberculosis in five young infants with nursery exposure: clinical, radiographic and CT findings. Pediatr Radiol 1998; 28(11): 836-40. doi:10.1007/s002470050477

8. NSW Health. Public hospital nurses (state) award. Available at: http://www.health.nsw.gov.au/nursing/employment/nurse_ award_wage_rates_11.asp (Cited 27 July 2012).

9. NSW Health. NSW public health system staff specialists (state) award. Available at: http://www.health.nsw.gov.au/archive/ policies/ib/2011/pdf/IB2011_036.pdf (Cited 27 July 2012).

10. NSW Health. Health employees' pharmacists (state) award. Available at: http://www.health.nsw.gov.au/resources/jobs/ conditions/awards/pdf/hsu_he_pharmacists.pdf (Cited 27 July 2012).

11. Carr D. 2012 private whole of life fixed vehicle operating costs. NRMA Motoring \& Services. Available at: http://www. mynrma.com.au/images/Motoring-PDF/Car-Operating-CostsReport-Summary.pdf (Cited 27 July 2012).

12. Department of Health and Ageing. Medicare benefits schedule book 2012. Canberra: DOHA; 2011.

13. Borgia P, Cambieri A, Chini F, Coltella L, Delogu G, Di Rosa E et al. Suspected transmission of tuberculosis in a maternity ward from a smear-positive nurse: preliminary results of clinical evaluations and testing of neonates potentially exposed, Rome, Italy, 1 January to 28 July 2011. Euro Surveill 2011; 16(40): 19984.

14. Sen M, Gregson D, Lewis J. Neonatal exposure to active pulmonary tuberculosis in a health care professional. CMAJ 2005; 172(11): 1453-6. doi:10.1503/cmaj.1031052

15. Burk JR, Bahar D, Wolf FS, Greene J, Bailey WC. Nursery exposure of 528 newborns to a nurse with pulmonary tuberculosis. South Med J 1978; 71(1): 7-10. doi:10.1097/00007611197801000-00004

16. Steiner P, Rao M, Victoria MS, Rudolph N, Buynoski G. Miliary tuberculosis in two infants after nursery exposure: epidemiologic, clinical and laboratory findings. Am Rev Respir Dis 1976; 113(3): 267-71.

17. Lonie C, Levy M, Frommer M. Counting the costs: a review of two tuberculosis mass contact screening investigations. $N S W$ Public Health Bull 1994; 5(2): 13-5. doi:10.1071/NB94008 